\title{
Neutron decay of the isobaric analog state in ${ }^{208} \mathrm{Bi}$
}

\author{
J.A. Bordewijk ${ }^{a, 1}$, A. Balanda ${ }^{b}$, D. Beaumel ${ }^{\text {c }}$, J. Blomgren $^{d, 2}$,
} S. Brandenburg ${ }^{a}$, G. van 't Hof ${ }^{\mathrm{e}}$, M.N. Harakeh ${ }^{\mathrm{e}, 3}$, M.A. Hofstee ${ }^{\mathrm{a}, 4}$, J. Jänecke ${ }^{f}$, A. Krasznahorkay ${ }^{g}$, H. Laurent ${ }^{c}$, L. Nilsson ${ }^{h}$, N. Olsson ${ }^{i}$, R. Perrino ${ }^{j}$, R. Siebelink ${ }^{k, 5}$, P.O. Söderman ${ }^{d}$, S.Y. van der Werf ${ }^{a, 6}$, A. van der Woude ${ }^{\text {a }}$

a Kernfysisch Versneller Instituut, Zernikelaan 25, 9747 AA Groningen. The Netherlands

b Jagiellonian University, Cracow, Poland

c Institut de Physique Nucléaire, Orsay, France

d Department of Radiation Sciences, Uppsala University, Box 535, S-75121 Uppsala, Sweden

e Faculteit Natuurkunde en Sterrenkunde, Vrije Universtiteit, de Boelelaan 1081, 1081 HV Amsterdam, The Netherlands

Department of Physics, University of Michigan, Ann Arbor, MI 48109, USA

g Institute of Nuclear Research of the Hungarian Academy of Sciences, H-4001 Debrecen, P.O. Box 51, Hungary

h The Svedberg Laboratory, Uppsala University, Box 533, S-75121 Uppsala, Sweden

' Department of Neutron Research, Uppsala University, Box 535, S-75121 Uppsala, Sweden

'Dipartimento di Fisica dell' Università and INFN, sezione di Lecce, 1-73100 Lecce, Italy

k Instituut voor Kern-en Stralingsfysica, Leuven, Belgium

Received 18 January 1993

\begin{abstract}
The isospin-forbidden neutron decay of the isobaric analog state in ${ }^{208} \mathrm{Bi}$ has been measured, following its excitation via the ${ }^{208} \mathrm{~Pb}\left({ }^{3} \mathrm{He}, \mathrm{t}\right){ }^{208} \mathrm{Bi}$ reaction at $61.2 \mathrm{MeV}$. In contrast to the proton decay, which is direct, the neutron decay spectrum has a statistical shape. Its branching ratio is determined to be $(37 \pm 3) \%$. The results suggest that the damping of the isospin impurity is fast. Therefore, neutron decay results mostly from the final stage of the damping process and does not contribute to the width of the IAS.
\end{abstract}

Key words: NUCLEAR REACTIONS: ${ }^{208} \mathrm{~Pb}\left({ }^{3} \mathrm{He}, \mathrm{t}\right){ }^{208} \mathrm{Bi}, E=61.2 \mathrm{MeV}$; magnetic spectrograph, neutron time-of-flight multi-detector; measured neutron decay; deduced neutron spectrum from IAS decay and branching ratio; statistical-decay calculations; isospin impurity. 


\section{Introduction}

The natural line width, $\Gamma$, of the isobaric analog state (IAS) $\left(T_{0}=(N-Z) / 2, T_{z}=\right.$ $T_{0}-1$ ) increases through the table of isotopes from around $20 \mathrm{keV}$ in the tin region to almost $350 \mathrm{keV}$ for the heaviest actinides [1].

If isospin were a good quantum number, all energetically allowed neutron decay would require a change in isospin of $\Delta T=\frac{3}{2}$ and would therefore be forbidden. Several channels for the decay of the IAS in ${ }^{208} \mathrm{Bi}$ are open for emission of light nuclei up to ${ }^{16} \mathrm{O}$. Some of these are again isospin forbidden and all are severely inhibited by the Coulomb barrier.

Proton decay into low-lying neutron-hole states relative to the parent nucleus, on the other hand, is isospin allowed. It has been shown to be a direct decay process and the decay rate is completely determined by the single-particle decay width and the neutron spectroscopic factor [2]. Thus, neglecting the gamma branch, proton emission would account for essentially all decay of the IAS in the case of good isospin.

Experimentally, however, one finds that the proton-decay branch amounts to 20$70 \%$ [1] and for ${ }^{208} \mathrm{Bi}$, studied in this work, its value is $(64 \pm 3) \%$ [2]. Thus one is led to conclude that either the IAS must have an admixture of $T_{<}=T_{0}-1$, which can be schematically expressed as:

$$
\left.|\mathrm{IAS}\rangle=\sqrt{1-\epsilon^{2}} T_{-} \mid \text {g.s. }\right\rangle+\epsilon\left|T_{<}\right\rangle,
$$

or that the quasi-continuum of nearby states has an admixture of isospin $T_{0}$ or both.

It is through such an isospin impurity that the IAS acquires a spreading width by damping over underlying states and that neutron decay and, in the actinides, even fission [3] become possible. The amplitudes of isospin admixtures are small. Proton decay is slowed down by the Coulomb barrier and, in heavy nuclei, must proceed through tunneling. Anticipating that the missing decay proceeds through neutron emission, one infers from the comparable magnitudes of the proton and neutron branches and from their single-particle transmission coefficients that the square of the admixed amplitude, $\epsilon^{2}$, is on the $1 \%$ level. Thus, the hindrance of the proton branch acts as a magnifying glass through which the isospin-forbidden neutron decay can be studied.

We report in this work on the first direct observation of the neutron-decay channel of the IAS. The interest is twofold. In the first place one wants to verify that the neutron branch indeed accounts for all of the "missing" decay. Second, and more important, one wants to measure the spectral shape which contains information about the time scale on which the isospin impurity in the IAS wave function damps out over configurations of higher complexity. In particular, it may answer the question whether neutron decay has

1 Present address: Rijksinstituut voor Volksgezondheid en Milieuhygiëne, Bilthoven, The Netherlands.

2 Present address: Department of Neutron Research, Uppsala University, Box 535, S-75121 Uppsala, Sweden.

3 Present address: Kernfysisch Versneller Instituut, 9747 AA Groningen, The Netherlands.

4 Present address: University of North Carolina, Chapel Hill, NC, USA.

5 Present address: Instituut voor Theoretische Fysica, Universiteit Leuven, Belgium.

6 Correspondence to: S.Y. van der Werf, KVI, Zernikelaan 25, 9747 AA Groningen, The Netherlands. 
an escape width. In this case it would contribute to the natural line width of the IAS in addition to the proton-decay escape width and the spreading width. Otherwise it is to be considered as a de-excitation in the final stage of the damping process. Since any contribution to the natural line width is, through the uncertainty principle, connected with the partial life time of a process that depletes the initial configuration, neutron decay will not contribute to the width in the latter case.

\section{Experimental techniques}

The charge-exchange reaction ${ }^{208} \mathrm{~Pb}\left({ }^{3} \mathrm{He}, \mathrm{t}\right){ }^{208} \mathrm{Bi}$ was used to excite the IAS in ${ }^{208} \mathrm{Bi}$. A $61.2 \mathrm{MeV}$ momentum-analysed ${ }^{3} \mathrm{He}$ beam was obtained from the AVF cyclotron of the KVI. Tritons were detected in the QMG/2 magnetic spectrograph [4], equipped with a multi-wire drift-chamber focal-plane detector [5]. The spectrograph was set at $0^{\circ}$ where the differential cross section of the IAS has its maximum. The magnetically less rigid ${ }^{3} \mathrm{He}$ beam was stopped inside the first dipolc magnet. Apart from the tritons, only deuterons and singly charged ${ }^{3} \mathrm{He}$ arrived at the detector. Clean particle identification

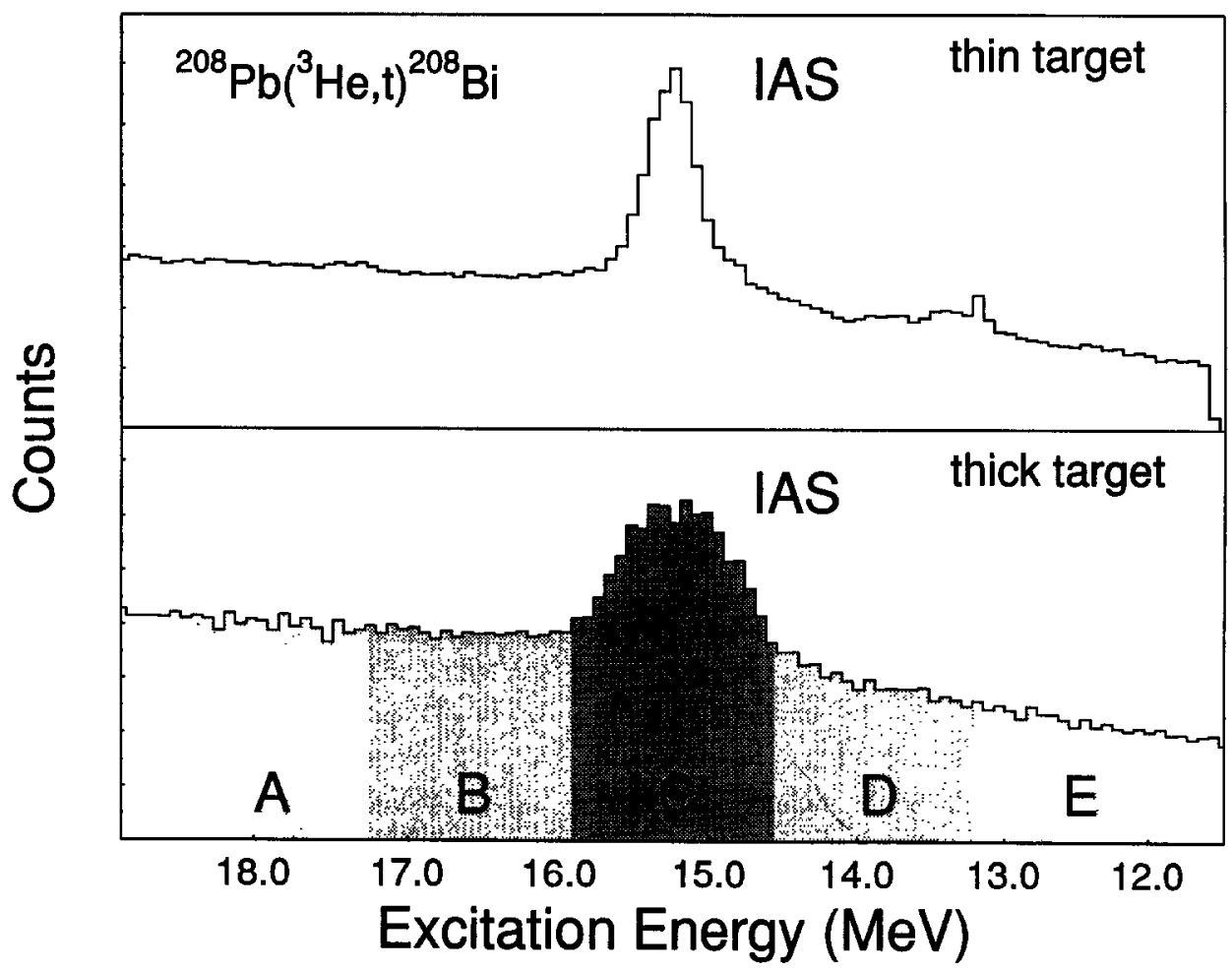

Fig. 1. Inclusive triton spectra from the ${ }^{208} \mathrm{~Pb}\left({ }^{3} \mathrm{He}, \mathrm{t}\right)$ reaction at $61.2 \mathrm{MeV}$ taken with a $5.4 \mathrm{mg} / \mathrm{cm}^{2}$ target (top) and an $18.6 \mathrm{mg} / \mathrm{cm}^{2}$ target (bottom). The shaded areas A through $\mathrm{E}$ indicate the $1.4 \mathrm{MeV}$ wide intervals for which neutron spectra were projected. 


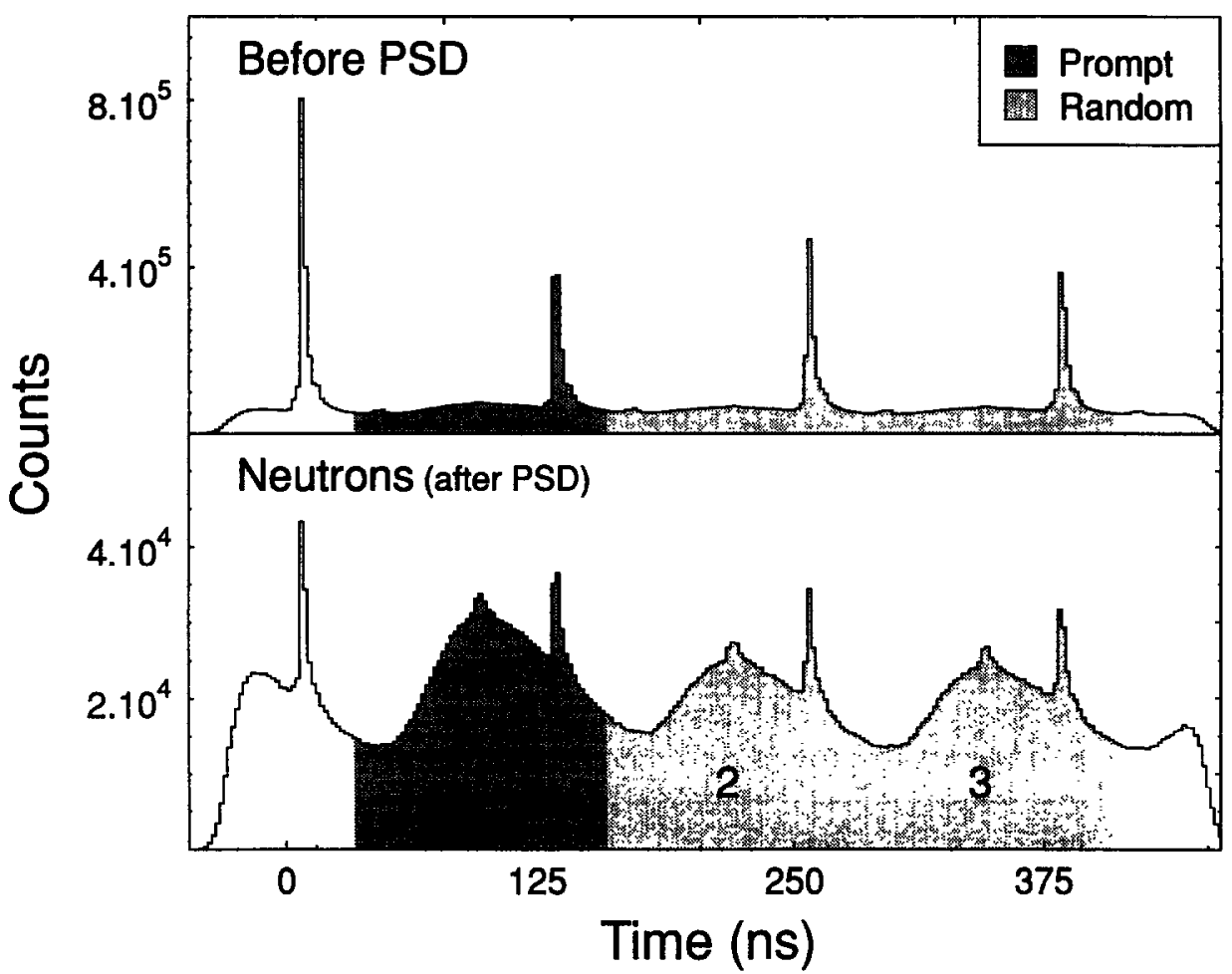

Fig. 2. Time spectra obtained with and without gamma suppression through pulse-shape discrimination (PSD). The dark- and light-shaded areas indicate the adopted prompt and random intervals. The prompt interval corresponds to the range $0.6-9.5 \mathrm{MeV}$ in neutron energy. The gamma contribution disappears in the prompt-random subtraction.

was achieved by making two-dimensional cuts in the plot of the position along the focal plane versus the energy deposit in the backing plastic detector. The thickness of the latter was just enough to stop the ${ }^{3} \mathrm{He}$ particles, but not the tritons and deuterons.

Fig. 1 shows triton spectra for a thin $\left(5.4 \mathrm{mg} / \mathrm{cm}^{2}\right)$ and a thick $\left(18.6 \mathrm{mg} / \mathrm{cm}^{2}\right)$ target.

The natural line width of the IAS in ${ }^{208} \mathrm{Bi}$ is $232 \mathrm{keV}$ [1]. In the present coincidence experiment the use of a thick target resulted in a broadening of the IAS to about $800 \mathrm{keV}$. This disadvantage is offset by an increase in the prompt-random ratio and the count rate.

Neutrons were detected in coincidence with tritons using the neutron time-of-flight (TOF) multi-detector setup EDEN [6]. For our experiment 39 detectors were used in the angular range $75^{\circ}-205^{\circ}$, each at a distance of $1.75 \mathrm{~m}$ from the target. The summed geometrical solid angle subtended by the neutron detectors was $398 \pm 16 \mathrm{msr}$. A figure showing the layout of the experiment can be found in Ref. [6] where the same experimental arrangement of the spectrograph and EDEN was used. More extensive details about the present experiment are given by Bordewijk [7].

The time interval between beam bursts was 125 ns. TOF spectra, with any of the 
neutron detectors as start and with the RF signal of the cyclotron as stop, are shown in Fig. 2, with and without gamma suppression based on pulse-shape discrimination.

The adopted prompt interval covers neutron energies up to $9.5 \mathrm{MeV}$ which is slightly above the maximum decay energy of the IAS $(8.28 \mathrm{MeV})$. This enforces a lower cutoff of $0.6 \mathrm{MeV}$ to avoid wrap-around. The width of the gamma flash is $2.4 \mathrm{~ns}$. This determines the energy resolution to be FWHM $=30$ and $400 \mathrm{keV}$ for 1 and $6 \mathrm{MeV}$ neutrons, respectively.

\section{Results}

The efficiency curve measured by Laurent et al. [6] for a threshold of $60 \mathrm{keV}$ deviates somewhat from that obtained with the Kent State University code [8]. We have applied the same threshold of $60 \mathrm{keV}$ in our data reduction and used the efficiency curve of Ref. [6].

Since the IAS has $I^{\pi}=0^{+}$, its decay is isotropic and neutron energy spectra were obtained by summing those from all the 39 identical detectors. Spectra corrected for random coincidences are shown in Fig. 3 for the five triton energy intervals indicated in Fig. 1 (bottom). The middle interval, C, contains $90 \%$ of the IAS. All spectra appear to be essentially of statistical shape.

\subsection{Statistical-model calculations}

Calculations have been performed with the statistical-model code CASCADE $[9,10]$. The neutron optical potentials of Rapaport et al. [11] were used to obtain the transmission coefficients.

$\mathrm{Up}$ to an excitation energy of $1 \mathrm{MeV}$ in the final nuclei ${ }^{207} \mathrm{Bi}$ and ${ }^{206} \mathrm{Bi}$ the known levels and their spins were used for the calculations. At higher energies the level densities were derived from the back-shifted Fermi-gas formula. Values for the level-density parameters $d a=A / a$ and $\Delta$ have been tabulated by Dilg et al. [12]. The value of $\Delta$ is less critical for the spectrum shape and has been fixed at $0.2 \mathrm{MeV}$.

Fig. 4 shows that all tabulated values of $d a$ for odd-odd, odd-even and even-even nuclei appear to cluster along the same "universal" band when plotted versus the quantity $N_{\mathrm{ph}}$, defined as the sum of the numbers of particles and holes away from the closed $N=126, Z=82$ configuration. Using this definition, ${ }^{207} \mathrm{Bi}$ and ${ }^{206} \mathrm{Bi}$ have $N_{\mathrm{ph}}=3$ and 4 , respectively, and a value for $d a$ around $18 \mathrm{MeV}$ seems appropriate for both nuclei.

A much better fit of the neutron evaporation spectra is, however, obtained for a $d a$ $=11 \mathrm{MeV}$, adopted for both ${ }^{207} \mathrm{Bi}$ and ${ }^{206} \mathrm{Bi}$. Calculated evaporation spectra are shown in Fig. 3 for both values of $d a$. These curves represent the calculated neutron spectra, multiplied by the detector efficiency [6] and folded with the energy resolution. The curves for $d a=11 \mathrm{MeV}$ reproduce the experimental spectra for all five intervals A-E very well. 


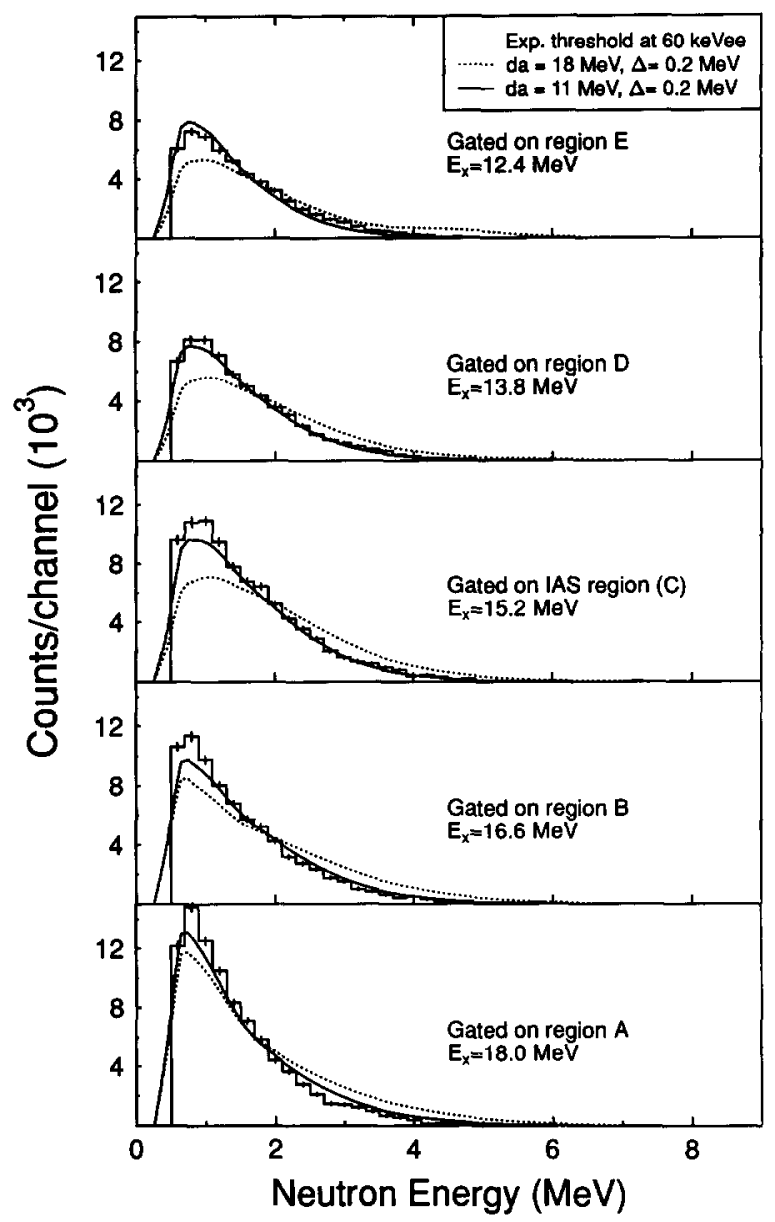

Fig. 3. Neutron spectra (histograms) for the five excitation energy intervals A-E, obtained by prompt-random sublraction. The solid and the dotted curves indicate calculations with the statistical-model code CASCADE, multiplied with the detector efficiency of Ref. [6] and folded with the energy resolution.

\subsection{Neutron spectrum of IAS}

The neutron spectrum from the decay of the IAS has been obtained by subtracting the estimated continuum contribution from the spectrum of interval $\mathrm{C}$. This estimate is based on a least-squares fit of the contents of the five intervals with a background that is assumed to follow a straight line and with the contributions from the IAS distributed over the five intervals as A: $1 \%, \mathrm{~B}: 4 \%, \mathrm{C}: 90 \%, \mathrm{D}: 4 \%$ and E: $1 \%$. These ratios follow from a convolution of the lorentzian line shape of the IAS with its width of $232 \mathrm{keV}$ and a gaussian smearing function, adjusted to reproduce the observed width in the singles spectrum (Fig. 1). For the intervals A and B the 2n-decay channel is open, causing an enhancement of the corresponding spectra at low neutron energies, compared to those from intervals C-E. When these $2 \mathrm{n}$ contributions are corrected for channel by channel 


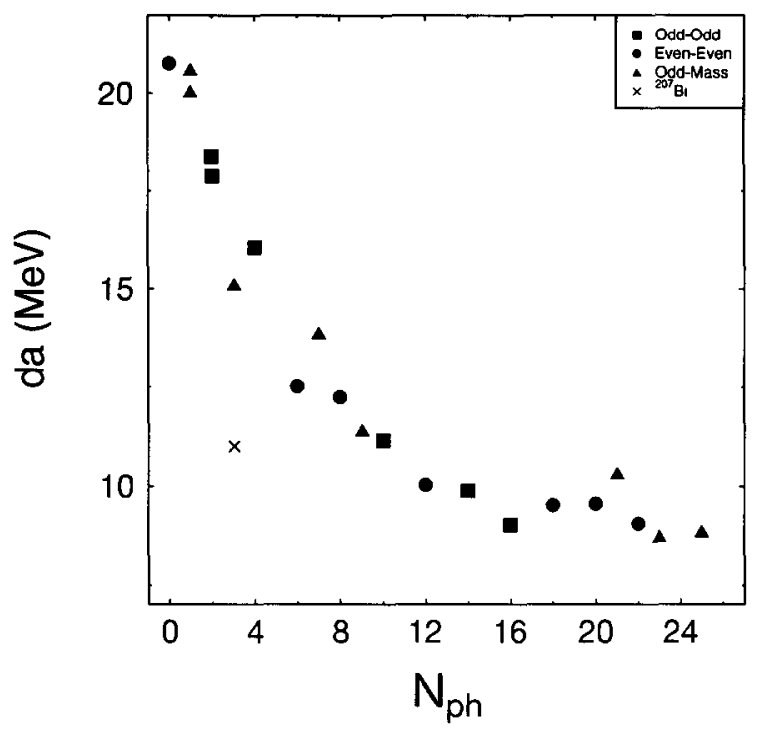

Fig. 4. Systematics of the level-density parameter $d a=A / a$ as function of $N_{\mathrm{ph}}$, defined as the sum of the number of nucleons (particles or holes) away from the shell closure at $Z=82, N=126$. The data points are from Dilg et al. [12]. The point with $d a=11 \mathrm{MeV}$ for ${ }^{207} \mathrm{Bi}$, which gives the best fit to our data, is indicated by a cross.

in a ratio that is derived from the CASCADE calculation, one finds that the $1 \mathrm{n}$ spectra for the intervals A, B, D and E vary only little. This justifies adopting their weighted average for the background in $\mathrm{C}$. The resulting spectrum for the decay of the IAS in interval $\mathrm{C}$ is shown in Fig. 5. It has again a statistical shape and curves representing

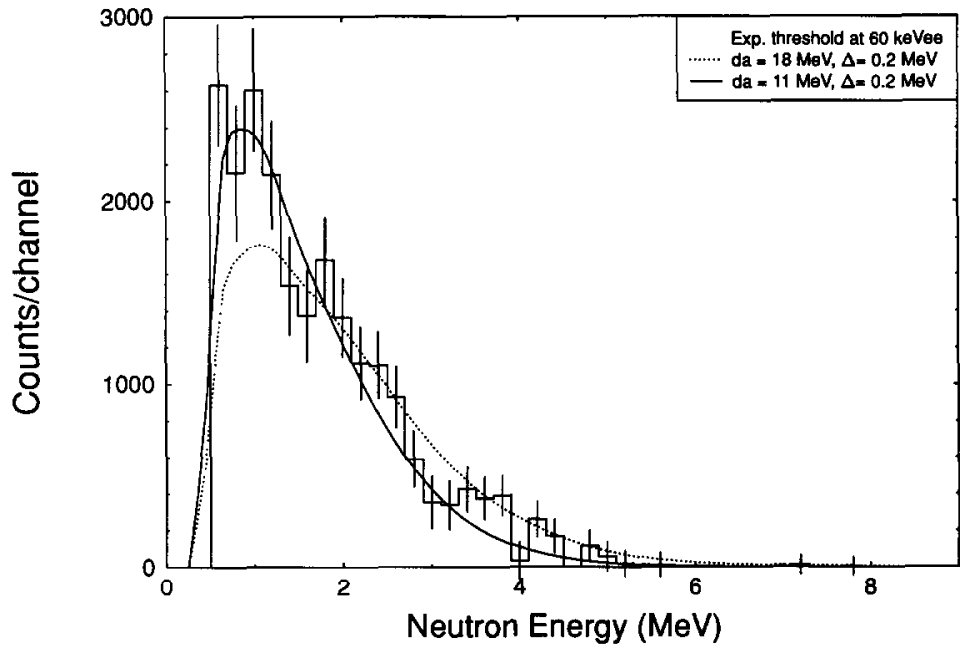

Fig. 5. Neutron spectrum from the decay of the IAS, obtained by subtraction from the spectrum of interval $\mathrm{C}$ the weighted average of the intervals $\mathrm{A}, \mathrm{B}, \mathrm{D}$ and $\mathrm{E}$ as described in the text. The solid and dashed curves represent calculations with the statistical-model code CASCADE, multiplied by the detector efficiency of Ref. [6] and folded with the energy resolution. 
CASCADE calculations with $d a=18$ and $11 \mathrm{MeV}$ are included.

\subsection{Neutron branching ratios of IAS and continuum}

Assuming isotropic emission of the neutrons, the neutron branching ratios for the intervals A-E and for the IAS are given, by:

$$
P^{\downarrow}=4 \pi \frac{\mathrm{d}^{2} \sigma / \mathrm{d} \Omega_{\mathrm{t}} \mathrm{d} \Omega_{\mathrm{n}}}{\mathrm{d} \sigma / \mathrm{d} \Omega_{\mathrm{l}}} .
$$

From the efficiency-corrected total number of coincidence counts, $N_{\mathrm{c}}$, and the singles counts, $\mathrm{N}_{\mathrm{s}}$, recorded in down-scaled mode, the branching ratio can be obtained from

$$
P^{\downarrow}=\frac{4 \pi}{\Omega_{\mathrm{EDEN}}} \frac{N_{\mathrm{c}}}{N_{\mathrm{s}}}
$$

The experimental neutron spectra were found to be well reproduced by a statisticalmodel calculation with $d a=11 \mathrm{MeV}$ after multiplication with the detector efficiency and folding with the energy resolution (Fig. 3). The true number of events in the neutron spectra, $N_{\mathfrak{c}}$, must account for the neutrons below the adopted cut-off energy of $600 \mathrm{keV}$. They have been obtained for each interval by integrating the CASCADE spectrum, normalized to the data.

Because the neutron detectors are arranged evenly over almost exactly half of a full circle, the assumption of isotropy is not even necessary. The branching ratio of the IAS is determined to be $(37 \pm 3) \%$. The background has a significantly higher neutron branching of about $70 \%$ for all intervals. This higher neutron branching is understood, because all excitations in ${ }^{208} \mathrm{Bi}$ in this region, other than the IAS, have isospin $\left(T_{0}-1\right)$ and will therefore favour the neutron channel. From an experiment at $81 \mathrm{MeV}$, slightly higher than in the present work, the Gamow-Teller Resonance has been estimated [13] to account for about one third of the continuum cross section in the region near the IAS [13]. Part of the inclusive $\left({ }^{3} \mathrm{He}, \mathrm{t}\right)$ cross section has been shown to stem from a quasifree pickup-breakup process [14], where the triton is accompanied by a fast proton. This process excites mostly low-lying particle-stable states in ${ }^{207} \mathrm{~Pb}$. Our result indicates that the pickup-breakup cross section amounts to about $30 \%$ of the inclusive cross section in the energy region around the IAS.

\section{Discussion and conclusions}

\subsection{Isospin mixing in a schematic model}

Any nuclear state $\left|a\left(T, T_{z}\right)\right\rangle$ acquires an isospin impurity through the Coulomb interaction. In a schematic model, comprehensively described by Auerbach [15], the effect is cast in the form of mixing with one single idealized state, the isovector monopole resonance, built upon the state $a$ : 


$$
\left|M\left(T^{\prime}, T_{z}\right)\right\rangle=\frac{1}{\sqrt{\mathcal{N}}}\left|T^{\prime}, T_{z}\right\rangle\left\langle T^{\prime}, T_{z}\left|\sum r^{2}(i) t_{z}(i)\right| a\left(T, T_{z}\right)\right\rangle .
$$

Here $\mathcal{N}$ is a normalization constant. The derivations given in Ref. [15] are restricted to the ground state, but are valid without change to any state, by virtue of the Brink hypothesis [16]. Using the notation of Ref. [15] the amplitude of the induced component $\epsilon_{T^{\prime}}$ in Eq. (1) is, in first-order perturbation theory, given by

$$
\epsilon_{T^{\prime}}=\frac{Z e^{2}}{2 R^{3}}\left\langle T, T_{z}, 1,0 \mid T^{\prime}, T_{z}\right\rangle \frac{h^{T^{\prime}}}{\left(E_{M_{T^{\prime}}}-E_{a}\right)},
$$

where $h^{T^{\prime}}$ is a reduced matrix element. Taking $a$ to be the IAS, one has ( $T=T_{0}=$ $\left.(N-Z) / 2, T_{z}=T_{0}-1\right), N$ and $Z$ referring to the target ground state and one finds

$$
\epsilon_{T-1}=\frac{Z e^{2}}{2 R^{3}} \sqrt{\frac{2 T-1}{T(2 T+1)}} \frac{h^{T-1}}{\left(E_{M_{T-1}}-E_{a}\right)},
$$

with $h^{T-1} \simeq \sqrt{\frac{2}{15}} A^{2 / 3} N^{1 / 2}$.

The identical expression is obtained by evaluating the mixing amplitude of the IAS with the $T_{0}-1$ component of the charge-exchange isovector monopole resonance,

$$
\begin{aligned}
M(T-1, T-1)= & \frac{1}{\sqrt{\mathcal{N}}}|T-1, T-1\rangle \\
& \times\left\langle T-1, T-1\left|\sum r^{2}(i) \tau_{-}(i)\right| T, T\right\rangle .
\end{aligned}
$$

Although the latter is not identical with the $T_{0}-1$ component of the IVM built upon the IAS, the isospin impurity of the IAS is usually discussed as an effect of the chargeexchange IVM $[17,15,18,19,1]$.

In the same way any of the underlying quasi-continuum $\left(T_{0}-1\right)$ states, $\mathrm{c}$, acquires an admixture of isospin $T_{0}$ through its "own" IVM, that is built upon it:

$$
\epsilon_{T}=\frac{Z e^{2}}{2 R^{3}} \frac{1}{\sqrt{T}} \frac{h^{T}}{\left(E_{M_{\mathrm{c}, T}}-E_{\mathrm{c}}\right)}
$$

where $h^{T} \simeq \sqrt{\frac{2}{15}} A^{2 / 3} Z^{1 / 2}$.

The energy splitting in the denominator is estimated as $\Delta E=\left(155 A^{-1 / 3} \pm 55 T / A\right)$ $\mathrm{MeV}$ [1]. Here the - sign in the Lane potential applies to the case of the IAS that acquires a component of lower isospin. The "grass" states receive a term of higher isospin and the Lane potential is positive in this case.

For heavy nuclei ( $T$ large), the ratio between the squares of the $T_{0}$ amplitude in the "grass" states and the $T_{0}-1$ amplitude in IAS is just $Z / N$, when the Lane potential is neglected and smaller when it is taken into account. Evaluating both admixtures with the above expressions ${ }^{7}$, one estimates for ${ }^{208} \mathrm{Bi}$ a squared amplitude $\epsilon^{2} \simeq 0.03$ for the IAS and $\simeq 0.02$ for the underlying states.

\footnotetext{
7 The expression of Ref. [15], Eq. (56) for the Coulomb matrix element contains an error and should be reduced by a factor 5 .
} 


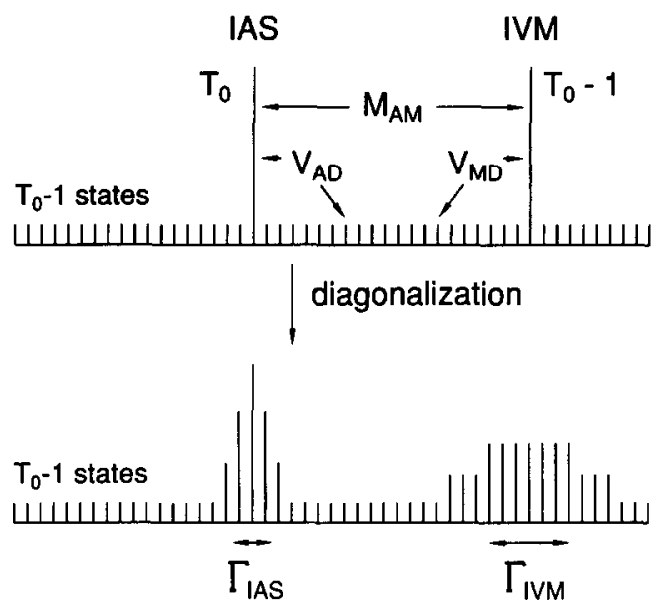

Fig. 6. Picket-fence model, illustrating the mixing of the IVM $\left(T_{0}-1\right)$ component into the IAS via the Coulomb force and the spreading over the quasi-continuum through a random matrix element.

Schematically the spreading width of the IAS can be explained in a picket-fence model $[1,20]$, illustrated in Fig. 6, where the IAS and the IVM $\left(T_{0}-1\right)$ mix through their Coulomb matrix element and where a small random matrix element $v_{\mathrm{MD}}$ connects the IVM to the $T_{0}-1$ "grass" states, c. In addition, the IAS can spread over the states $\mathrm{c}$, also with a random matrix element $v_{\mathrm{AD}}$, through their $T_{0}$ components. This time-independent picture involves a full diagonalization of this problem and is usually replaced by two separate diagonalizations, resulting in an expression for the spreading width [19]

$$
S(E)=\frac{1}{2 \pi} \frac{\Gamma(E)}{\left(E-E_{\mathrm{IVM}}-\Delta_{\mathrm{IVM}}(E)\right)^{2}+\left(\frac{1}{2} \Gamma(E)\right)^{2}} .
$$

Compared with the usual treatment [19] in which only the spreading route via a $\left(T_{0}-1\right)$ IVM admixture to the "grass" is considcred, the "local" sprcading width, $\Gamma(E)$, must be generalized to include also the route via the induced isospin impurity of the "grass" states. This will not change the form of the strength function in Eq. (9). It is not clear how the relative magnitude of the random matrix elements $v_{\mathrm{MD}}$ and $v_{\mathrm{AD}}$, which connect to the "grass" states, must be estimated. Under the assumption that they are equal, the effect will, neglecting the Lane potential, lead to a multiplication by a factor $A / N$ of the usual estimate for the first route:

$$
\Gamma(E) \simeq \frac{A}{N} \Gamma_{\mathrm{IVM}}(E)
$$

\subsection{Time development of the isospin impurity and decay modes}

It appears from the present work that the neutron decay of the IAS is fully statistical whereas the proton decay is direct. This result suggests that the time development of the IAS follows a hierarchy of exciton states as depicted in Fig. 7. 


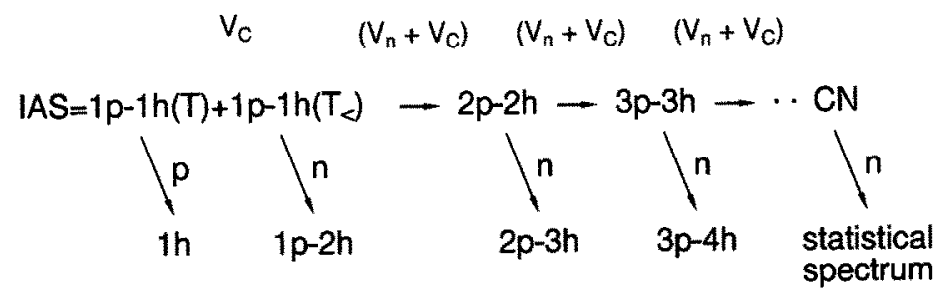

Fig. 7. Schematic representation of the time evolution and decay modes of the IAS.

The proton decay stems from the normal isospin $\left(T_{0}\right)$ component of the IAS, which according to Eq. (6), has a squared amplitude $\left(1-\epsilon^{2}\right)$ of about 0.97 . The admixed $1 \mathrm{plh}$ $\left(T_{0}-1\right)$ IVM component, with a squared amplitude of $\epsilon^{2} \approx 0.03$ can mix with nearby states, or alternatively the $1 \mathrm{plh} T_{0}$ IAS component can mix with them through their own isospin impurity. The subsequent time development then proceeds via an exciton hierarchy of $n \mathrm{p} n \mathrm{~h}$ configurations towards the compound nucleus (C.N.).

At each stage of this chain neutron decay competes with the next step of the damping process. Of particular interest is the possibility of a semi-direct emission from the $1 \mathrm{p} 1 \mathrm{~h}$ stage where, in analogy to the electronic Auger effect, a strongly interacting pair of neutrons splits up. One of them fills the neutron hole that was produced in the reaction, while the other escapes into the continuum. The signature of this process would be neutron decay into the low-lying seniority $=1$ states in ${ }^{207} \mathrm{Bi}$ if the neutrons formed a Cooper pair. In the neutron-decay spectrum of the IAS, Fig. 5, this would correspond to energies between $7 \mathrm{MeV}\left(E_{\mathrm{x}}=1.28 \mathrm{MeV}\right)$ and $8.28 \mathrm{MeV}$ (decay to the ground state). For neutrons that did not initially occupy time-reversed orbits, the Auger decay from the first stage will lead to seniority $=3$ states and its contribution to the neutron decay spectrum of the IAS is expected in the neutron-energy range 4-7 MeV.

Experimentally, no enhancement over a statistical spectrum shape is ohserved in the region where this nuclear Auger effect might show up. We interpret this fact as evidence that the damping process is fast compared to the decay branching out of the earlier $n \mathrm{p}(n+1) \mathrm{h}$ stages of the exciton chain.

In this picture the so-called "never-come-back" hypothesis is implied. The spreading width is determined by the partial level density of the $2 \mathrm{p} 2 \mathrm{~h}$ configurations, which act as doorway states. The approximate $\left(E_{\mathrm{x}}\right)^{3}$ dependence of the spreading width of the IAS noted in Refs. $[21,1,20]$ is consistent with this hypothesis as indeed, in an equidistant-spacing model with level spacing $g$, this partial level density is given by $\rho_{2 \mathrm{p} 2 \mathrm{~h}} \propto g\left(g E_{\mathrm{x}}\right)^{3}[22]$.

\subsection{Statistical-model calculations including isospin}

The statistical-model code CASCADE has the option [10] to treat isospin selection rules rigorously. Using this option, Fig. 8 shows two statistical decay spectra. The hypothetical case of unmixed isospin (bottom), where $\epsilon^{2}=0$, is shown in the lower spectrum. It features the primary proton and gamma decay of the IAS. Neutron decay is forbidden. The top spectrum of Fig. 8 is still a hypothetical spectrum with a very 


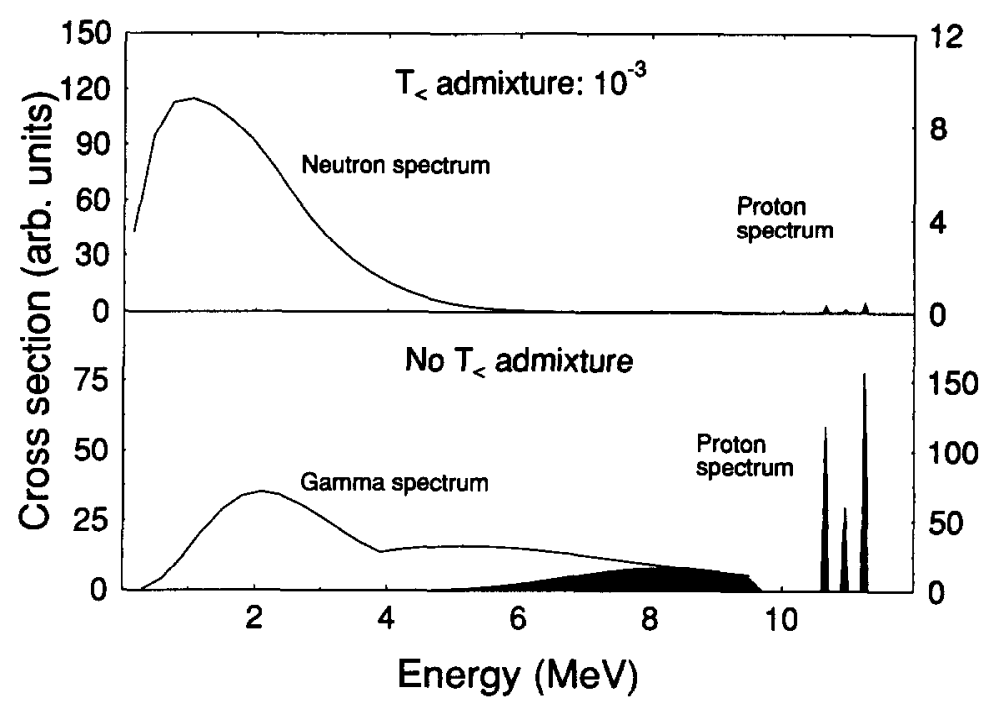

Fig. 8. Statistical decay spectra for the hypothetical cases of unmixed isospin (bottom) and very small isospin admixture $\epsilon^{2}=10^{-3}$ (top) in the IAS wave function. The vertical scales at the right-hand side refer to the proton spectra, those at the left side to the gamma spectrum (bottom) and the neutron spectrum (top).

small value $\epsilon^{2}=10^{-3}$ of a $\left(T_{0}-1\right)$ component in the IAS. This tiny admixture, at least ten times smaller than estimated in the present work, appears sufficient to bring the neutron-decay branch for statistical decay above $99 \%$. It is therefore evident that the observed proton decay, with a branching ratio of $(64 \pm 3) \%$ cannot be a statistical process, but must be a direct decay.

\subsection{Level density parameters}

A remarkable result of the present work, and puzzling at first sight, is the small value $d a=11 \mathrm{MeV}$ that is required to describe the neutron evaporation spectra, where a value of about $18 \mathrm{MeV}$ is indicated by the systematics as compiled by Dilg et al. [12] The tabulated values have, however, been obtained from low-energy neutron resonances and apply therefore to a region of excitation energy just above the neutron-separation threshold. Their extrapolation to the excitation energy of the IAS must be considered with some caution.

Another source of information on the level densities that applies to the IAS region is provided by the spreading widths. As discussed in Subsect. 4.2, these scale with the partial 2p2h level density $\rho_{2 \mathrm{p} 2 \mathrm{~h}} \propto g\left(g E_{\mathrm{x}}\right)^{3}$. Here $g=\left(6 / \pi^{2}\right) a=\left(6 / \pi^{2}\right) A / d a[22]$. The decrease in the tabulated level-density parameter $a$ by almost a factor 2 around the double shell closure at ${ }^{208} \mathrm{~Pb}$ would, by virtue of the factor $g^{4}$ in the expression for $\rho_{2 \mathrm{p} 2 \mathrm{~h}}$, be magnified to a dramatic dip of a factor 16 in the systematics of the spreading width. Instead, no significant deviation is observed from the general smooth increase with mass and excitation energy $[1,20]$. This observation has earlier been interpreted by Jänecke et al. [1] as evidence that near the IAS excitation energy values of $d a$ of 
about $10.5-11.0$ are required in the $\mathrm{Pb}$ region.

\subsection{Conclusions}

The neutron branching ratio of the IAS decay has been determined to be $(37 \pm 3) \%$ and accounts, within the error, for essentially all the "missing" decay other than the isospin-allowed proton branch, which had been found earlier to be $(64 \pm 3) \%$ [2].

The results of the present work confirm the hypothesis that neutron decay results from the final stage of the exciton hierarchy. It has no significant escape width. Neutron decay does therefore not give a contribution to the total width of the IAS. This point of view is strengthened by the interesting case of the light $\mathrm{Sb}$ isotopes, where the energy of the IAS is below the threshold for neutron instability. Nevertheless, the data on the spreading width follow the same global trend as function of mass, charge and excitation energy as in nuclei where the IAS is neutron unstable [23].

As indeed neutron decay appears not to add to the width, direct proton decay is the only contributor to the escape width, $\Gamma^{\dagger}$.

Escape and spreading widths are through the uncertainty principle to be interpreted as inverse partial life times of the initial configuration and are therefore additive. This justifies a posteriori the usual procedure $[1,21,20,23]$ of deriving experimentally the spreading width as the difference between the total width and the proton escape width:

$$
\Gamma^{\downarrow}=\Gamma_{\text {tot }}-\Gamma^{\dagger} \text {. }
$$

\section{Acknowledgement}

This work was done as part of the research program of the "Stichting voor Fundamenteel Onderzoek der Materie" (FOM) with financial support from the "Nederlandse Organisatie voor Wetenschappelijk Onderzoek" (NWO).

\section{References}

[1] J. Jänecke, M.N. Harakeh and S.Y. van der Werf, Nucl. Phys. A 463 (1987) 571, and references quoted therein.

[2] S.Y. van der Werf, M.N. Harakeh and E.N.M. Quint, Phys. Lett. B 216 (1989) 15, and references quoted therein.

[3] S.Y. van der Werf, N. Blasi, S. Brandenburg, A.G. Drentje, M.N. Harakeh, W.A. Sterrenburg, B. Visscher, A. van der Woude, R. De Leo and H. Janszen, Phys. Lett. B 105 (1981) 111.

[4] A.G. Drentje, H.A. Enge and S.B. Kowalski, Nucl. Instr. Meth. 161 (1974) 485.

[5] J.M. Schippers, W.T.A. Borghols and S.Y. van der Werf, Nucl. Instr. Meth. A 247 (1986) 467.

[6] H. Laurent, H. Lefort, D. Beaumel, Y. Blumenfeld, S. Fortier, S. Galès, J. Guillot, J.C. Roynette, P. Volkov and S. Brandenburg, Nucl. Instr. Meth. A 326 (1993) 517.

[7] J.A. Bordewijk, Ph.D. thesis, Rijksuniversiteit Groningen, 1993 (unpublished).

[8] R.A. Cecil, B.D. Anderson and K. Madey, Nucl. Instr. Meth. 161 (1979) 439.

[9] F. Pühlhofer, Nucl. Phys. A 208 (1977) 267.

[10] M.N. Harakeh, Program CASCADE, extended version (1992) unpublished. 
[11] J. Rapaport, V. Kulkarni and R.W. Finlay, Nucl. Phys. A 330 (1979) 15.

[12] W. Dilg, W. Schantl, H. Vonach and M. Uhl, Nucl. Phys. A 217 (1973) 269.

[13] C. Gaarde, J.S. Larsen, A.G. Drentje, M.N. Harakeh and S.Y. van der Werf, Phys. Rev. Lett. 46 (1981) 902.

[14] E.H.L. Aarts, R.K. Bhowmik, R.J. de Meijer and S.Y. van der Werf, Phys. Lett. B 102 (1981) 307.

[15] N. Auerbach, Nucl. Phys. A 182 (1972) 247.

[16] D. Brink, Ph.D. thesis, Oxford University (1955) unpublished.

[17] A.Z. Mekjian, Phys. Rev. Lett. 25 (1970) 888.

[18] N. Auerbach, Proc. 1980 RCNP Int. Symp. on highly excited states in nuclear reactions, Osaka, Japan (1980) p. 653.

[19] W.M. MacDonald and M.C. Birse, Phys. Rev. C 29 (1984) 425.

[20] S.Y. van der Werf, Proc. 6th Int. Conf. on nuclear reaction mechanisms, ed. E. Gadioli, Varenna, Italy (1991) p. 530.

[21] H.J. Hofmann, S. Brandenburg, P. Grasdijk, M.N. Harakeh, W.A. Sterrenburg and S.Y. van der Werf, Nucl. Phys. A 433 (1985) 181.

[22] T. Ericson, Adv. Phys. 9 (1960) 425.

[23] J. Jänecke, J.A. Bordewijk, S.Y. van der Werf and M.N. Harakeh, Nucl. Phys. A 552 (1993) 323. 